\section{Performance of the QuantiF- ERON-TB Gold In-Tube test to Monitor Treatment of Active Pulmonary Tuberculosis in Taiwan}

\section{Kow Tong Chen ${ }^{1,2}$, Pin Hui Wang ${ }^{2}$, Shun Tien Chien ${ }^{3}$ and Shou Chien Chen ${ }^{4 *}$}

${ }^{1}$ Department of Occupational Medicine, Tainan Municipal Hospital, Tainan, Taiwan

${ }^{2}$ Department of Public Health, College of Medicine, National Cheng Kung University, Tainan, Taiwan

${ }^{3}$ Chest Hospital, Ministry of Welfare and Health, Tainan, Taiwan

${ }^{4}$ Department of Family Medicine, Da-Chien General Hospital, Miaoli, Taiwan

\begin{abstract}
\section{Background}

Timely and effective monitoring of Tuberculosis (TB) treatment is an important strategy for prevention and control of TB. The aims of this study were to assess the performance of the QuantiFERON-TB Gold In-Tube (QFT-GIT) in diagnosis and monitoring response to anti-tuberculosis treatment in patients with active Pulmonary Tuberculosis (PTB).

\section{Methods}

We conducted a retrospective case-control study. Between March and September 2014, 28 cases with active PTB and 28 controls with no mycobacterial infection, matched by age within 3 years and week of visit to Taiwan Chest Hospital, were enrolled in the study. Serial testing by QFT-GIT at baseline and after 2 months of treatment was performed. A comparison of the performance of QFT-GIT with that of sputum culture status among study subjects was conducted.

\section{Results}

Compared to baseline, $25(89 \%)$ cases had a decline in Interferon-gamma (IFN- $\gamma$ ) responses at 2 months culture-positive and the end of an intensive phase of anti-tuberculosis treatment, whereas three did not. Their IFN- $y$ responses declined significantly from baseline to 2 months (medium 2.11 vs. $0.88 ; P<0.005$ ). The sensitivity of the QFT-GIT test for detection of pulmonary
\end{abstract}

*Corresponding author: Shou Chien Chen, Department of Family Medicine, Da Chien General Hospital, Miaoli, Taiwan, Tel: +886 3735125; Email: scchen818@yahoo.com.tw

Citation: Chen KT, Wang PH, Chien ST, Chen SC (2016) Performance of the QuantiFERON-TB Gold In-Tube test to Monitor Treatment of Active Pulmonary Tuberculosis in Taiwan. J Community Med Public Health Care 3: 022.

Received: August 03, 2016; Accepted: September 28, 2016; Published: October 13, 2016 tuberculosis at cut-off points of $0.35 \mathrm{IU} / \mathrm{ml}, 0.20 \mathrm{IU} / \mathrm{ml}$ and $0.10 \mathrm{IU} / \mathrm{ml}$ was $71.4 \%, 78.6 \%$ and $82.1 \%$ respectively. The specificity at cut-off points of $0.35 \mathrm{IU} / \mathrm{ml}, 0.20 \mathrm{IU} / \mathrm{ml}$ and $0.10 \mathrm{IU} / \mathrm{ml}$ was $64.3 \%, 57.1 \%$, and $53.6 \%$ respectively. The Positive Predictive Value (PPV) at cutoff points of $0.35 \mathrm{IU} / \mathrm{ml}, 0.20 \mathrm{IU} / \mathrm{ml}$ and $0.10 \mathrm{IU} / \mathrm{ml}$ was $66.7 \%, 64.7 \%$ and $63.9 \%$, respectively.

\section{Conclusion}

Although our study indicates that QFT-GIT has moderate sensitivity and specificity, our results support the candidate of QFT-GIT assay as a potential tool for the diagnosis of tuberculosis and monitoring the efficacy of anti-tuberculosis treatment.

Keywords: Pulmonary tuberculosis; QuantiFERON-TB Gold In-Tube test; Sensitivity; Specificity

\section{Introduction}

Tuberculosis (TB) is still an important public health problem throughout the world. In 2013, approximately 9.0 million people developed TB, with 1.5 million deaths [1]. However, early diagnosis of TB remains a complicated issue in the control and prevention of TB. Today, Tuberculin Skin Tests (TST) is one of the most commonly used methods for diagnosis of TB due to its low cost and convenience in most countries. However, there are several disadvantages to this method of TB diagnosis, such as poor specificity in people with Bacille Calmette-Guerin (BCG) vaccination and infection with Non-Tuberculous Mycobacteria (NTM), low sensitivity in immunocompromised persons and the requirement of two clinical visits to read the results $[2,3]$. Interferon gamma (IFN- $\gamma$ ) Release Assays (IGRAs) detect the ex vivo release of the key anti-tuberculosis cytokine, IFN- $\gamma$ [3]. Previous studies have demonstrated that IGRAs may be an alternative for diagnosis of TB [4]. IGRAs include proteins that are more unique and specific to Mycobacterium tuberculosis (M. tuberculosis) than those in the Purified Derivative (PPD) and encoded by genes located in the Region of Difference 1 (RD 1) within the M. tuberculosis genome. These genes are not found in M. bovis BCG or most environmental Mycobacteria [5]. The QuantiFERON-TB Gold In-Tube test (QFT-GIT) assay (Cellestis, Carniege, Victoria, Australia) measures the IFN- $\gamma$ concentration in whole blood after stimulation by specific tuberculosis antigens (e.g., Early Secreted Antigenic Target-6 [ESAT-6], Culture Filtrate Protein-10 [CFP-10]) and TB7.7 antigen [6,7]. It is recognized as an efficient alternative test to detect the presence of Latent $\mathrm{Myco}$ bacterium Tuberculosis Infection (LTBI) [6-9]. Whether the QFT-GIT will be useful in monitoring responses to anti-tuberculosis treatment is unclear $[7,10]$. The potential prognostic use of IFN- $\gamma$ responses has been studied in research describing Isoniazid (INH) treatment of LTBI and anti-tuberculosis treatment of active tuberculosis. In the case of LTBI, the prognostic use of IFN- $\gamma$ is not yet clearly established. It has been reported that the IFN- $\gamma$ responses after INH prophylaxis may be stronger [11], persistent [12], decreased [13], or dependent on the antigen used $[14,15]$. Similarly, in the treatment of active TB, some studies have observed post-treatment mitigation of the IFN- $\gamma$ response [16-18], while others have reported persistent or even stronger IFN- $\gamma$ responses after anti-tuberculosis treatment [19-22]. There is limited information regarding the effectiveness of the QFT-GIT test in Taiwan. The purpose of this study was to assess the performance of 
QFT-GIT test for diagnosis and monitoring in the treatment of active $\mathrm{TB}$ in Taiwan.

\section{Background}

The study was conducted in the Tainan Chest Hospital of the Ministry of Welfare and Health in Taiwan. Tainan Chest Hospital provides respiratory disease services comprising voluntary counseling and testing, medical care and laboratory testing. More than 4,320 people with respiratory disorder visit this hospital each year. Of these, 300 (7\%) were diagnosed with tuberculosis.

\section{Materials and Methods}

\section{Cases}

Cases were identified as 28 consecutive patients presenting to Chest Hospital of the Ministry of Welfare and health between March and September 2014 with active Pulmonary Tuberculosis (PTB). Active PTB cases were defined as patients with a history of cough for more than 3 weeks and positive culture for Mycobacterium tuberculosis (M. tuberculosis) complex in $\geqq 1$ sputum sample [23]. If both M. tuberculosis complex and Non-Tuberculosis Mycobacteria (NTM) were identified, we classified the subjects as active PTB. Participants were classified as NTM culture-positive when NTM was isolated in $\geqq 1$ sputum sample. The remaining participants were classified as having no mycobacterial infection. In this study, we assessed the clinical characteristics and outcomes among cases with active PTB when compared with those of the participants classified as having no mycobacterial infection. Exclusion criteria were age $<18$ years, pregnancy, positive Human Immunodeficiency Virus (HIV) serology, use of immunosuppressive drugs and previous history of anti-tuberculosis treatment. Informed consent was obtained before sample collection. Patients who agreed to participate in the study and were willing to attend regular follow-up visits were asked to complete a questionnaire that included limited demographic information and symptoms. Participants provided first spot and second early morning sputum specimens for the laboratory study of M. tuberculosis. Laboratory testing of the sputum was performed by the Laboratory Division of Chest Hospital.

\section{Controls}

Controls were retrospectively selected from medical records. Patients presenting to the Chest Hospital in the same weeks as cases and having respiratory-related illness but no Mycobacteria in sputum culture were enrolled to controls. Controls were individually matched to cases patients by age within three years and interviewed.

\section{Study procedures}

All the study patients underwent QFT-GIT assay, chest X-ray examination and sputum culture at baseline and 2 months. All patients enrolled in the study received a standardized anti-tuberculosis treatment including daily INH, Rifampicin (RMP), Ethambutol (EMB) and Pyrazinamide (PZA) for 2 months, with follow-up at the end of 2 months of treatment. Patients who remained sputum culture-positive at the end of 2 months were given INH, RMP, PZA and EMB (HRZE) for 1 month; if they still remained culture-positive at the end of 3 months, they were excluded from the study and treated according to their culture and drug sensitivity patterns.

Approval for the study was obtained from the Institutional Review Board for the Protection of Human Subjects at Tainan Chest Hospital and National Cheng Kung University Hospital, Taiwan.

\section{Laboratory tests}

Sputum samples were decontaminated and centrifuged and the pellet was used to prepare smears that were examined microscopically and graded for acid-fast bacilli using the Ziehl-Neelsen and fluorescent microscopy of Auramine O staining methods. Decontaminated specimens were inoculated in BACTEC 7H9 liquid medium for the isolation and identification of $M$. tuberculosis. The QFT-GIT test was conducted on $1 \mathrm{ml}$ of venous blood incubated at $37^{\circ} \mathrm{C}$ for $16-24 \mathrm{~h}$. These procedures have been described elsewhere [24,25]. Calculations were performed using the QFT-GIT analysis software provided by manufacturer. QFT-GIT was considered positive if the estimated IFN- $\gamma$ concentration in the sample exceeded the negative control by 0.35 International Units (IU) $/ \mathrm{ml}$. All IFN- $\gamma$ concentration values in the subsequent analyses represent the difference between samples and negative controls.

Additional specimens from 10 participants were sent to the Taiwan Centers for Disease Control (Taiwan CDC) for laboratory test replication (QFT); agreement between the results from the Taiwan CDC and Tainan Municipal Hospital was good.

\section{Statistical analysis}

Mean and standard deviations were used to describe the distribution of the concentration variables. The prognostic performance of QFT-GIT was examined using the parameters of a diagnostic test-sensitivity, specificity, Predictive Positive Value (PPV). Chi-square/Fisher's exact tests and t-tests were used to analyze the differences in the distribution of study subjects by IFN- $\gamma$ response (failing vs. persistent or stronger) at 2 months. Significance of the derived P-values was defined as an alpha level of 0.05 or less. All the analyses were conducted using Stata 9.0 (Stata Corp, College Station, TX, USA).

\section{Definition}

Sensitivity was defined as the proportion of people with the disease who had a positive test for the disease. Specificity was defined the proportion of people without the disease who had a negative test for the disease. Positive predictive value was defined as the probability of disease in a patient with a positive test result. Negative predictive value was defined as the probability of not having the disease when the test result was negative. The predictive value was determined by the sensitivity and specificity of the test and the prevalence of disease in the population being tested. The predictive value is regarded to be the most relevant characteristic in clinician interpretation of test results [3].

\section{Results}

Table 1 displays the demographic characteristics of study subjects. During the study period, a total of 64 cases were identified as eligible study subjects. Of these, $56(87.5 \%)$ met the inclusion criteria and were enrolled in the study. The mean age of study subjects was 59.3 years ( $\mathrm{SD}=15.7$ years); 38 (67.9\%) were male; thirty-nine $(69.6 \%)$ of study subjects had received BCG vaccination and thirty-four (60.7\%) were positive by QFT-GIT at baseline. Compared with controls, cases had higher rates of diabetes $(\mathrm{P}=0.02)$ and QFT-GIT test positivity $(\mathrm{P}=0.007)$. Other variables were not significantly different between the patients with culture-negativity and patients with culture-positivity $(\mathrm{P}>0.05$ in all).

Figure 1 illustrates the production of IFN- $\gamma$ at baseline and 2 months at cut-off point of $0.35 \mathrm{IU} / \mathrm{ml}$. IFN $-\gamma$ responses declined significantly from baseline to 2 months (medium $2.11 \mathrm{IU} / \mathrm{ml}$ vs. 0.88 


\begin{tabular}{|c|c|c|c|c|}
\hline \multirow{2}{*}{ Variables } & Total & Cases & Controls & \multirow{2}{*}{ P-value } \\
\hline & $N=56$ & $N=28$ & $\mathrm{~N}=\mathbf{2 8}$ & \\
\hline $\begin{array}{c}\text { Age (Yr) } \\
\text { (Mean } \pm S D)\end{array}$ & $59.3 \pm 15.7$ & $56.4 \pm 16.0$ & $61.9 \pm 15.4$ & 0.19 \\
\hline \multicolumn{5}{|l|}{ Sex } \\
\hline Male & 38 & 18 & 20 & \multirow{2}{*}{0.64} \\
\hline Female & 18 & 10 & 8 & \\
\hline \multicolumn{5}{|c|}{ BCG vaccination } \\
\hline Yes & 39 & 19 & 20 & \multirow{2}{*}{0.60} \\
\hline No & 17 & 9 & 8 & \\
\hline \multicolumn{5}{|l|}{ QFT-GIT test } \\
\hline Positive & 34 & 20 & 10 & \multirow{2}{*}{0.007} \\
\hline Negative & 22 & 8 & 18 & \\
\hline \multicolumn{5}{|l|}{ Symptoms } \\
\hline \multicolumn{5}{|l|}{ Cough } \\
\hline Yes & 47 & 22 & 25 & \multirow{2}{*}{0.50} \\
\hline No & 9 & 6 & 3 & \\
\hline \multicolumn{5}{|c|}{ Loss of weight } \\
\hline Yes & 26 & 14 & 12 & \multirow{2}{*}{0.61} \\
\hline No & 30 & 14 & 16 & \\
\hline \multicolumn{5}{|l|}{ Night sweats } \\
\hline Yes & 5 & 3 & 2 & \multirow{2}{*}{0.64} \\
\hline No & 51 & 25 & 26 & \\
\hline \multicolumn{5}{|c|}{ Co-morbidities } \\
\hline \multicolumn{5}{|l|}{ CVDs } \\
\hline Yes & 5 & 3 & 2 & \multirow{2}{*}{0.66} \\
\hline No & 51 & 25 & 26 & \\
\hline \multicolumn{5}{|l|}{ Diabetes } \\
\hline Yes & 14 & 11 & 3 & \multirow{2}{*}{0.02} \\
\hline No & 42 & 17 & 25 & \\
\hline \multicolumn{5}{|c|}{ Family history of TB } \\
\hline Yes & 8 & 3 & 5 & \multirow{2}{*}{0.48} \\
\hline No & 48 & 25 & 23 & \\
\hline
\end{tabular}

Table 1: Demographic characteristics of study subjects in Chest Hospital, Taiwan.

${ }^{*}$ Comparison of cases to controls.

$\mathrm{IU} / \mathrm{ml} ; \mathrm{P}<0.005)$. Compared to baseline, $25(89 \%)$ cases had a decline and $3(11 \%)$ had persistent Interferon-gamma (IFN- $\gamma$ ) responses at 2 months culture-positive and the end of an intensive phase of anti-tuberculosis treatment. Three (11\%) cases remained culture-positive at 2 months post-treatment.

\section{Performance of QFT-GIT test}

Table 2 displays the performance of the QFT-GIT test at different cut-off values for detecting active pulmonary tuberculosis. At a QFT-GIT cut-off value of $\geqq 0.35 \mathrm{IU} / \mathrm{ml}$, the sensitivity was $71.4 \%$, the specificity was $64.3 \%$ and the PPV was $66.7 \%$ for the QFT GIT test for PTB. At a QFT-GIT cut-off value of $\geqq 0.20 \mathrm{IU} / \mathrm{ml}$, the sensitivity was 78.6\%, specificity was $57.1 \%$ and PPV was $64.7 \%$ for the QFT-GIT test for PTB. At a QFT-GIT cut-off value of $\geqq 0.10 \mathrm{IU} / \mathrm{ml}$, the sensitivity was $82.1 \%$, specificity was $53.6 \%$ and PPV was $63.9 \%$ for the QFT-GIT test for PTB.

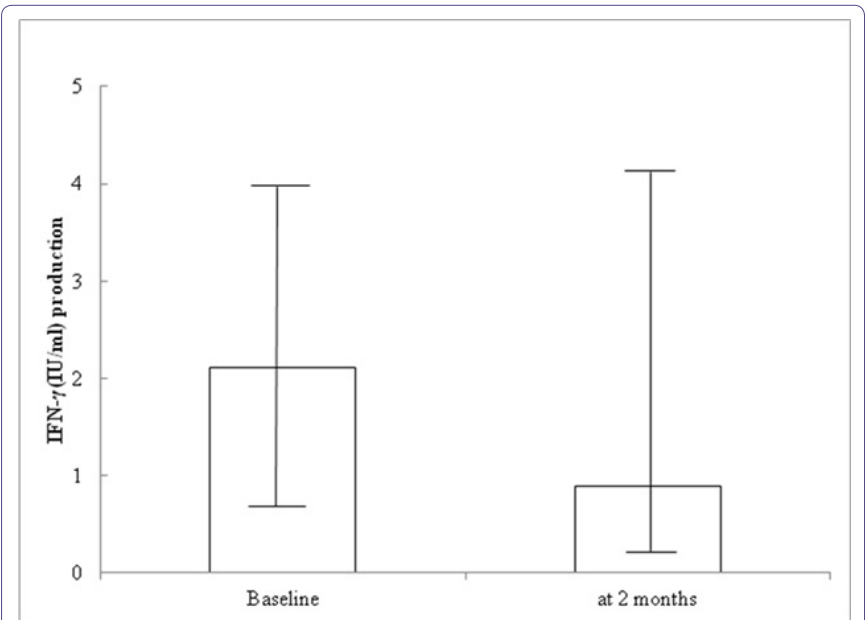

Figure 1: IFN-y production using serial QTF-GIT assays (at baseline and 2 months after treatment initiation) in subjects with active tuberculosis on a standard regimen $(n=28)$.

\begin{tabular}{|c|c|c|c|c|c|c|}
\hline \multirow{2}{*}{\multicolumn{2}{|c|}{$\begin{array}{l}\text { QFT-GIT Test Cut-Off } \\
\text { Value }(I \mathrm{I} / \mathrm{ml})\end{array}$}} & Cases & Controls & Sensitivity & Specificity & PPV \\
\hline & & $\mathrm{N}=28$ & $N=28$ & (\%) & $(\%)$ & (\%) \\
\hline \multirow{2}{*}{$\geqq 0.35$} & + & 20 & 10 & \multirow{2}{*}{71.4} & \multirow{2}{*}{64.3} & \multirow{2}{*}{66.7} \\
\hline & - & 8 & 18 & & & \\
\hline \multirow{2}{*}{$\geqq 0.20$} & + & 22 & 12 & \multirow{2}{*}{78.6} & \multirow{2}{*}{57.1} & \multirow{2}{*}{64.7} \\
\hline & - & 6 & 16 & & & \\
\hline \multirow{2}{*}{$\geqq 0.10$} & + & 23 & 21 & \multirow{2}{*}{82.1} & \multirow{2}{*}{53.6} & \multirow{2}{*}{63.9} \\
\hline & - & 5 & 7 & & & \\
\hline
\end{tabular}

Table 2: Performance of the QFT-GIT test in the detection of active PTB.

Note: QFT-GIT test: QuantiFERON-TB Gold In-Tube test; PTB: Pulmonary Tuberculosis; PPV: Predictive Positive Value.

\section{Discussion}

With the exception of studies from Japan [8], Korea [26] and India [27], most other published studies [28-32] have reported the QFTGIT assay to have moderate sensitivity (61-81\%). In this study, we had similar findings, with a sensitivity of $71.4 \%$ and specificity of $64.3 \%$ to detect active PTB identified at the baseline QFT-GIT assessment. These findings are important, as the accuracy of IFN- $\gamma$ responses has not been unequivocally established for the diagnosis of active TB. The previous study [33] shows the QFT-GIT has better performance than TST for the diagnosis of the tuberculosis. However, neither of them is stable in the diagnosis of TB.

Serial testing by QFT-GIT demonstrated an overall progressive weakening of the IFN- $\gamma$ response during anti-tuberculosis treatment, and QFT-GIT assessment after 2 months of treatment could be an independent and sensitive indicator of the likelihood of failing to convert sputum culture status. Our study showed $11 \%$ of study subjects were persistent IFN- $\gamma$ at 2 months culture-positive at the end of anti-tuberculosis treatment. A previous study [27] suggested that nearly half of the study cohort was still positive by QFT-GIT after 6 months of anti-tuberculosis treatment. In this study, we did not have data to follow-up after 6 months of anti-tuberculosis treatment. There are several possible explanations why immune responses to Even Specific Antigens (ESAT-6 and CFP-10) may not have dropped below pre-defined levels, resulting in positive tests after anti-tuberculosis treatment: 1) T-cell responses to ESAT-6 may persistent as a scar of previously treated or quiescent infection [21];2) the anti-tuberculosis 
treatment may only have helped infection revert to a stage of latency rather than conferring sterilizing immunity [34];3) it has been argued that in some individuals, a population of activated T-cells persists in the absence of direct mycobacterial antigen stimulation, even for several years after completing treatment [22]; 4) It is possible that a continued exposure to $M$. tuberculosis during anti-tuberculosis treatment, especially as the environmental burden is high and 5) there is inter-individual variation in the strength of the IFN- $\gamma$ response that can be partly explained by genetic polymorphisms in the host [35]. Although the IFN- $\gamma$ level measured by QFT-GIT assay decreased after successful anti-TB treatment in most patients, any of them exhibited QFT-GIT reversion to negativity. Thus, the reversion to negativity of QFT-GIT assay may not be a good surrogate for treatment response. Of course, the short follow-up time can affect.

The accuracy of the QFT-GIT assay varied according to the cut-off point. A cut-off of $0.35 \mathrm{IU} / \mathrm{ml}$ for diagnosis of active TB had moderate sensitivity (71.4\%) and specificity (64.3\%). If the cut-off point was set at $0.20 \mathrm{IU} / \mathrm{l}$, the sensitivity increased to $78.6 \%$, but the specificity decreased to $57.1 \%$. Similarly, if the cut-off point was at 0.10 $\mathrm{IU} / \mathrm{ml}$, the sensitivity increased to $82.1 \%$; however, the specificity was $53.6 \%$. Consequently, when using the QFT-GIT assay for monitoring response to treatment, it may be necessary to revise the cut-off to be prognostically meaningful. Future studies will need to address this issue more directly using larger numbers of patients treated for active TB.

There were several limitations in our study. First, the number of patients included in the study was small. However, this study provides important information regarding the role of QFT-GIT assays in the monitoring of active PTB treatment. Second, TST status may influence QFT-GIT results [36,37]. In our study, we did not evaluate the influence of TST status on the prognostic performance of QFT-GIT. Third, our study used mycobacterial culture as the gold standard for the diagnosis of TB. This methodology sometimes gives false negative results due to poor sputum sample collection or paucibacillary [38]. Therefore, our study may underestimate the performance of the QFT-GIT test for diagnosis of TB. Despite these limitations, our results support the utilization of the QFT-GIT assay as a potential tool to monitor the efficacy of anti-tuberculosis treatment in cases of active PTB.

In conclusion, our study indicates that QFT-GIT has moderate sensitivity and specificity; however, our results support the candidate of QFT-GIT assay as a potential tool for the diagnosis of tuberculosis and monitoring the efficacy of anti-tuberculosis treatment.

\section{Acknowledgement}

We are grateful to the staff of Tainan Chest Hospital, Ministry of Welfare and Health, Taiwan, for their skilled interviewing and collection of study information.

\section{References}

1. World Health Organization (2014) Global tuberculosis report 2014. World Health Organization, Geneva, Switzerland.

2. Detjen AK, Keil T, Roll S, Hauer B, Mauch H, et al. (2007) Interferon-gamma release assays improve the diagnosis of tuberculosis and nontuberculous mycobacterial disease in children in a country with a low incidence of tuberculosis. Clin Infect Dis 45: 322-328.

3. Davies PDO, Lalvani A, Thillai M, Gordon SB (2014) Clinical Tuberculosis $\left(5^{\text {th }}\right.$ edn), Taylor \& Francis Group, Abingdon, UK.
4. Lu P, Chen X, Zhu LM, Yang HT (2016) Interferon-Gamma Release Assays for the Diagnosis of Tuberculosis: A Systematic Review and Meta-analysis. Lung 194: 447-458.

5. Dilektasli AG, Erdem E, Durukan E, Eyüboğlu FÖ (2010) Is the T-cell-based interferon-gamma releasing assay feasible for diagnosis of latent tuberculosis infection in an intermediate tuberculosis-burden country? Jpn J Infect Dis 63: 433-436.

6. Carvalho AC, Pezzoli MC, El-Hamad I, Arce P, Bigoni S, et al. (2007) QuantiFERON-TB Gold test in the identification of latent tuberculosis infection in immigrants. J Infect 55: 164-168.

7. Dheda K, Pooran A, Pai M, Miller RF, Lesley K, et al. (2007) Interpretation of Mycobacterium tuberculosis antigen-specific IFN-gamma release assays (T-SPOT.TB) and factors that may modulate test results. J Infect 55: 169-173.

8. Kobashi Y, Obase Y, Fukuda M, Yoshida K, Miyashita N, et al. (2006) Clinical reevaluation of the QuantiFERON TB-2G test as a diagnostic method for differentiating active tuberculosis from nontuberculous mycobacteriosis. Clin Infect Dis 43: 1540-1546.

9. Lalvani $A$ (2007) Diagnosing tuberculosis infection in the 21st century: new tools to tackle an old enemy. Chest 131: 1898-1906.

10. Menzies D, Pai M, Comstock G (2007) Meta-analysis: new tests for the diagnosis of latent tuberculosis infection: areas of uncertainty and recommendations for research. Ann Intern Med 146: 340-354.

11. Wilkinson KA, Kon OM, Newton SM, Meintjes G, Davidson RN, et al. (2006) Effect of treatment of latent tuberculosis infection on the $T$ cell response to Mycobacterium tuberculosis antigens. J Infect Dis 193: 354-359.

12. Pai M, Joshi R, Dogra S, Mendiratta DK, Narang P, et al. (2006) Persistently elevated T cell interferon-gamma responses after treatment for latent tuberculosis infection among health care workers in India: a preliminary report. $J$ Occup Med Toxicol 1: 7.

13. Higuchi K, Harada N, Mori T (2008) Interferon-gamma responses after isoniazid chemotherapy for latent tuberculosis. Respirology 13: 468-472.

14. Chee CB, KhinMar KW, Gan SH, Barkham TM, Pushparani M, et al. (2007) Latent tuberculosis infection treatment and T-cell responses to Mycobacterium tuberculosis-specific antigens. Am J Respir Crit Care Med 175: 282-287.

15. Goletti D, Parracino MP, Butera O, Bizzoni F, Casetti R, et al. (2007) Isoniazid prophylaxis differently modulates T-cell responses to RD1-epitopes in contacts recently exposed to Mycobacterium tuberculosis: a pilot study. Respir Res 8: 5.

16. Lalvani A, Nagvenkar P, Udwadia Z, Pathan AA, Wilkinson KA, et al. (2001) Enumeration of $T$ cells specific for RD1-encoded antigens suggests a high prevalence of latent Mycobacterium tuberculosis infection in healthy urban Indians. J Infect Dis 183: 469-477.

17. Carrara S, Vincenti D, Petrosillo N, Amicosante M, Girardi E, et al. (2004) Use of a $\mathrm{T}$ cell-based assay for monitoring efficacy of antituberculosis therapy. Clin Infect Dis 38: 754-756.

18. Aiken AM, Hill PC, Fox A, McAdam KP, Jackson-Sillah D, et al. (2006) Reversion of the ELISPOT test after treatment in Gambian tuberculosis cases. BMC Infect Dis 6: 66 .

19. Ulrichs T, Anding R, Kaufmann SH, Munk ME (2000) Numbers of IFN-y-producing cells against ESAT- 6 increase in tuberculosis patients during chemotherapy. The International Journal of Tuberculosis and Lung Disease 4: 1181-1183.

20. Wu-Hsieh BA, Chen CK, Chang JH, Lai SY, Wu CH, et al. (2001) Long-lived immune response to early secretory antigenic target 6 in individuals who had recovered from tuberculosis. Clin Infect Dis 33: 1336-1340.

21. Chee CB, KhinMar KW, Gan SH, Barkham TM, Pushparani M, et al. (2007) Latent tuberculosis infection treatment and T-cell responses to Mycobacterium tuberculosis-specific antigens. Am J Respir Crit Care Med 175: 282-287.

22. Pai M, Joshi R, Bandyopadhyay M, Narang P, Dogra S, et al. (2007) Sensitivity of a whole-blood interferon-gamma assay among patients with pulmonary tuberculosis and variations in T-cell responses during anti-tuberculosis treatment. Infection 35: 98-103. 
23. Bjerrum S, Oliver-Commey J, Kenu E, Lartey M, Newman MJ, et al. (2016) Tuberculosis and non-tuberculous Mycobacteria among HIV-infected individuals in Ghana. Trop Med Int Health 21: 1181-1190.

24. Diel R, Loddenkemper R, Meywald-Walter K, Niemann S, Nienhaus A (2008) Predictive value of a whole-blood IFN-gamma assay for the development of active TB disease. Am J Respir Crit Care Med 177: 1164-1170.

25. Gerogianni I, Papala M, Klapsa D, Zinzaras E, Petinaki E, et al. (2008) Wholeblood interferon-gamma assay for the diagnosis of tuberculosis infection in an unselected Greek population. Respirology 13: 270-274.

26. Kang YA, Lee HW, Hwang SS, Um SW, Han SK, et al. (2007) Usefulness of whole-blood interferon-gamma assay and interferon-gamma enzyme-linked immunospot assay in the diagnosis of active pulmonary tuberculosis. Chest 132: 959-965.

27. Katiyar SK, Sampath A, Bihari S, Mamtani M, Kulkarni H (2008) Use of the QuantiFERON-TB Gold In-Tube test to monitor treatment efficacy in active pulmonary tuberculosis. Int J Tuberc Lung Dis 12: 1146-1152.

28. Dewan PK, Grinsdale J, Kawamura LM (2007) Low sensitivity of a wholeblood interferon-gamma release assay for detection of active tuberculosis. Clin Infect Dis 44: 69-73.

29. Goletti D, Carrara S, Vincenti D, Saltini C, Rizzi EB, et al. (2006) Accuracy of an immune diagnostic assay based on RD1 selected epitopes for active tuberculosis in a clinical setting: a pilot study. Clin Microbiol Infect 12: 544-550.

30. Sester M, Sotgiu G, Lange C, Giehl C, Girardi E, et al. (2011) Interferon- $\gamma$ release assays for the diagnosis of active tuberculosis: a systematic review and meta-analysis. Eur Respir J 37: 100-111.
31. Nishimura T, Hasegawa N, Mori M, Takebayashi T, Harada N, et al. (2008) Accuracy of an interferon-gamma release assay to detect active pulmonary and extra-pulmonary tuberculosis. Int J Tuberc Lung Dis 12: 269-274.

32. Vincenti D, Carrara S, Butera O, Bizzoni F, Casetti R, et al. (2007) Response to region of difference 1 (RD1) epitopes in human immunodeficiency virus (HIV)-infected individuals enrolled with suspected active tuberculosis: a pilot study. Clin Exp Immunol 150: 91-98.

33. Lu P, Chen X, Zhu LM, Yang HT (2016) Interferon-Gamma Release Assays for the Diagnosis of Tuberculosis: A Systematic Review and Meta-analysis. Lung 194: 447-458.

34. Nakielna EM, Cragg R, Grzybowski S (1975) Lifelong follow-up of inactive tuberculosis: its value and limitations. Am Rev Respir Dis 112: 765-772.

35. Sallakci N, Coskun M, Berber Z, Gürkan F, Kocamaz H, et al. (2007) Interferon-gamma gene+874T-A polymorphism is associated with tuberculosis and gamma interferon response. Tuberculosis (Edinb) 87: 225-230.

36. Igari H, Watanabe A, Sato T (2007) Booster phenomenon of QuantiFERON-TB Gold after prior intradermal PPD injection. Int J Tuberc Lung Dis 11: 788-791.

37. Leyten EM, Prins C, Bossink AW, Thijsen S, Ottenhoff TH, et al. (2007) Effect of tuberculin skin testing on a Mycobacterium tuberculosis-specific interferon-gamma assay. Eur Respir J 29: 1212-1216.

38. Ndzi EN, Nkenfou CN, Gwom LC, Fainguem N, Fokam J, et al. (2016) The pros and cons of the QuantiFERON test for the diagnosis of tuberculosis, prediction of disease progression, and treatment monitoring. International Journal of Mycobacteriology 5: 177-184. 